\title{
Research on Intelligent Control of NC WEDM Working Fluid
}

\author{
Xiaomei Jiang ${ }^{1, a}$, Zhengxiao Hua, b ${ }^{1, Y a n n i a n ~ R u i ~}{ }^{2, c}$ \\ ${ }^{1}$ School of Mechanical Engineering, Changshu Institute of Technology, Changshu, 215500, China \\ ${ }^{2}$ College of Mechatronics Engineering, Soochow University, Suzhou, 215021, China \\ ajszjjxm@163.com, ${ }^{b}$ hzx@cslg.edu.cn, ${ }^{b}$ ryn@suda.edu.cn
}

Keywords: WEDM; working fluid; fuzzy control; conductivity

\begin{abstract}
NC WEDM has played an increasingly important role in the automotive, aerospace and other precision machinery mold processing and gone towards the direction of high precision and efficiency. In the process, the conductivity and temperature of the working fluid affect discharge result and workpiece thermal deformation. There is ambiguity in the working fluid conductivity measurement and control process. Fuzzy variables are used to describe the conductivity of the working fluid and processing parameters to determine the conductivity range of the working fluid. Experiments show the working fluid fuzzy control can avoid the repeated action of actuator and improve the control reliability to ensure the accuracy of the system control.
\end{abstract}

\section{Introduction}

As an important auxiliary part of wire cutting, the working fluid control technology has been widely used in many domestic and foreign manufacturers of wire cutting machine tool, but on ways of improving their work efficiency and control methods did not receive the attention and focus. The control of WEDM working fluid includes two aspects, first, how to accurately determine the electrical conductivity of the working fluid, and then control the appropriate actuator to make the conductivity of the working fluid reach the set limits; Second, how to compensate the temperature of the working fluid and keep constant within a certain range[1, 2]. There are not many relative research reports on the combination of different processing modes and features to develop methods of intelligent control of conductivity, so this is an important issue in this paper.

Due to larger working fluid pool, there is lag in the conductivity control, and conductivity controlled in a certain point is unscientific, it will cause actuator a repeated action, so it is necessary to make the working fluid conductivity fuzzification. In addition, the processing material thicknesses, the thickness of the electrode wires and these parameters related to the conductivity of the working fluid also have ambiguity. Therefore, the fuzzy control of working fluid is a try. Fuzzy control implements the control law based on the knowledge (rules) and even semantic description and provides a relatively easy design method for the nonlinear controller, especially when the control object or process with uncertainties and difficult to use conventional linear control theory, it is more effective [3]. In this thesis, the expert fuzzy controller was adopted which contains strategic knowledge, it allows more complex hierarchical rules, and can determine the application which rules between low-level rules, namely: IF (process status 1) THEN (use rule set A); IF (process status N) THEN (use rule set B). All the rules described above are called "event-driven rules", processing in the so-called forward link mode; these rules can only be applied when state in the process "matching" with pre-determined conditions [4].

\section{The working fluid intelligent control program}

Fig. 1 is a diagram of the working fluid circulating intelligent control system in the wire cutting process. P1 pumps the suitable clean working fluid from the working fluid clear liquid tank to the nozzle for feed flow. The processed working fluid flows into the processing tank, and then recycles 


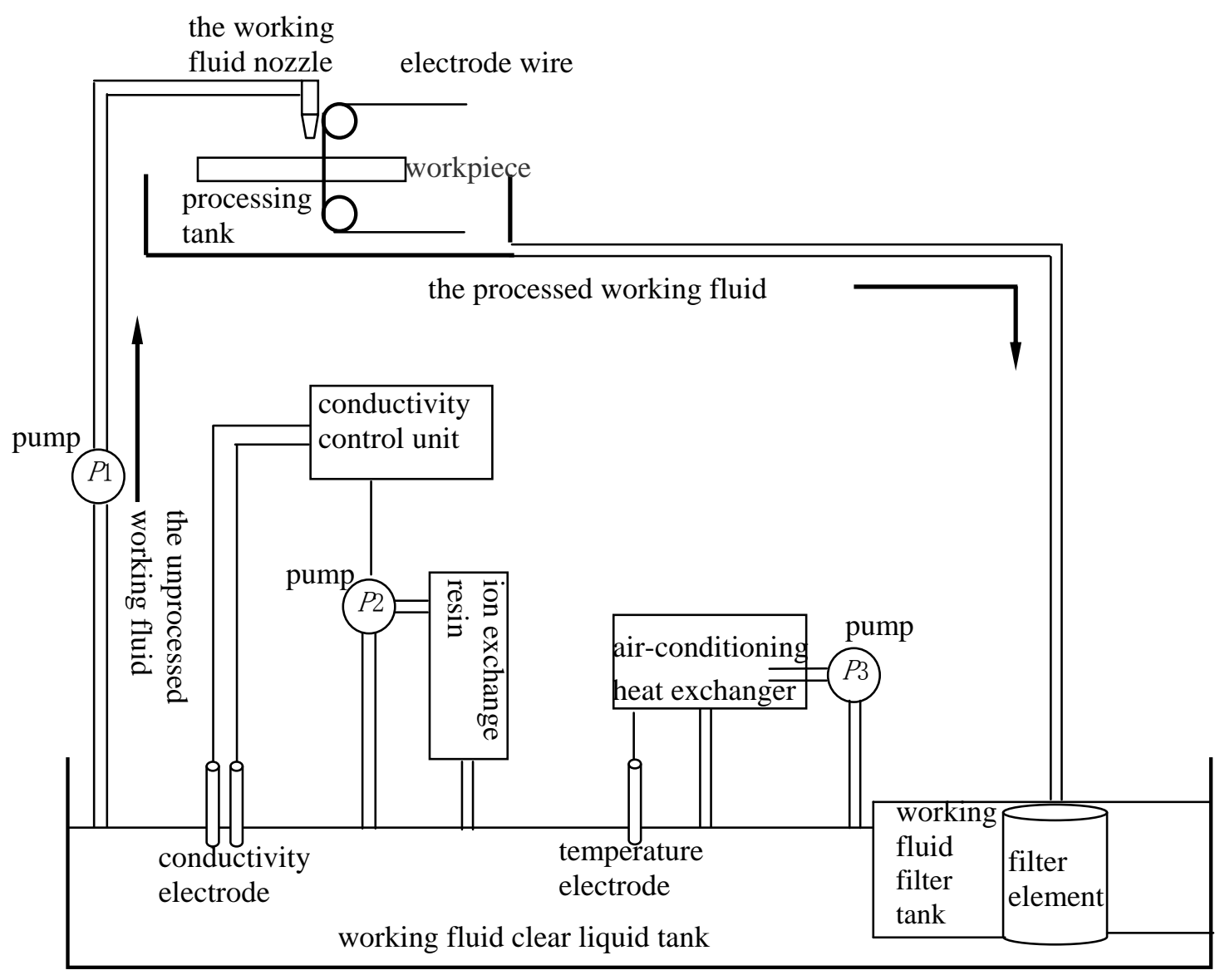

Fig. 1 The working fluid circulating control system of wire cutting processing

through the return line to the filter element of working fluid filtration tank, after filters out the debris, flows to the working fluid clear liquid tank, forms the working fluid processing cycle.

The conductivity control unit measures the conductivity of the working fluid by conductivity measurement electrode in the clear liquid tank, to get the voltage signal which is proportional to the conductivity of the working fluid, after amplification, rectification, filtering, anti-jamming transmission and optical isolation[5], into the conductivity controller, using the fuzzy intelligent control, if the electrical conductivity higher than required set point, start the pump P2, make the working fluid flow through the ion exchange resin, reducing the conductivity of the working fluid, and determine the effective state of ion resin through the rate of change of the working fluid conductivity error. Now the temperature measurement electrodes in the working fluid can measure the temperature of the working fluid, if the measured temperature is too high or low, air conditioning exchanger can start cooling or heating, and start P3, make the working fluid and air-conditioning exchange heat, and the working fluid temperature can be constant within the set range[6].

\section{The working fluid fuzzy control model}

Intelligent control system for the working fluid process is shown in Fig.1; the temperature control is fulfilled by air conditioning heat exchanger, independent of the conductivity control system and control easy. So this research focused on working fluid conductivity related parameters and settings fuzzification and fuzzy rule sets.

The settings of working fluid conductivity in the process is acquired by a series of fuzzy variables from the fuzzy control rules given, two main fuzzy variables including processing material thickness and electrode wire diameter. After filtering conductivity data collected, the error and error change rate of conductivity was obtained. Then through fuzzification, and comparison with the set value, ion 
resin exchange pump action was activated according to the fuzzy control model and process. The fuzzy control process was shown in Fig.2.

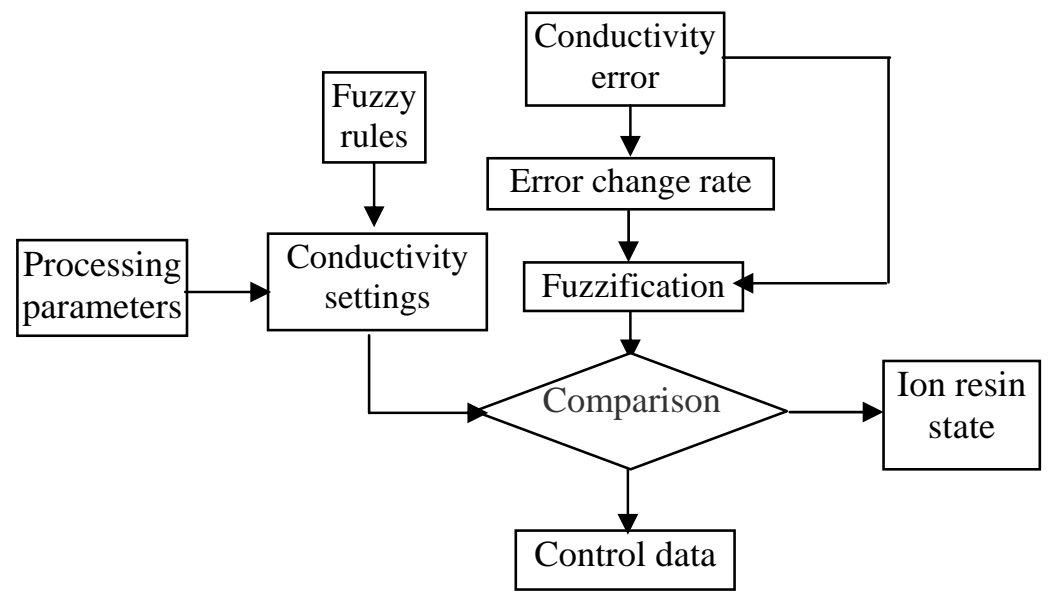

Fig. 2 Basic process of fuzzy control

Assuming $\mathrm{E}$ and $\mathrm{EC}$ be deviation and the deviation change rate the current measurement values and required values, taking the basic domain as follows: $E=\left[e_{\min }, e_{\max }\right], E C=\left[e c_{\min }, e c_{\max }\right]$. Quantify $\begin{aligned} E & \Rightarrow X=\left[x_{1}, x_{2}, \cdots x_{p_{1}}\right] \\ \text { its basic domain, } & \text {, After obtaining the quantitative domain, to define the }\end{aligned}$ fuzzy subset of each variable. $X=\left\{A_{i}\right\}_{(i=1,2, \cdots, n)} \quad Y=\left\{B_{j}\right\}_{(j=1,2, \cdots, m)}$. Where, $A_{i}$ and $B_{j}$ respectively the fuzzy subset of $X, Y$, can be represented by linguistic variables $P B, P M, \ldots N M, N B$, etc. The membership function can be obtained from membership function of each element in quantitative domain of each fuzzy subset elements. With $\mathrm{x}$, for example, the membership degree of the elements of $\mathrm{X}$ to $\left\{A_{i}\right\}$ is shown in Table 1 .

Table1 the membership degree of $x_{i}$ to $\left\{A_{i}\right\}$

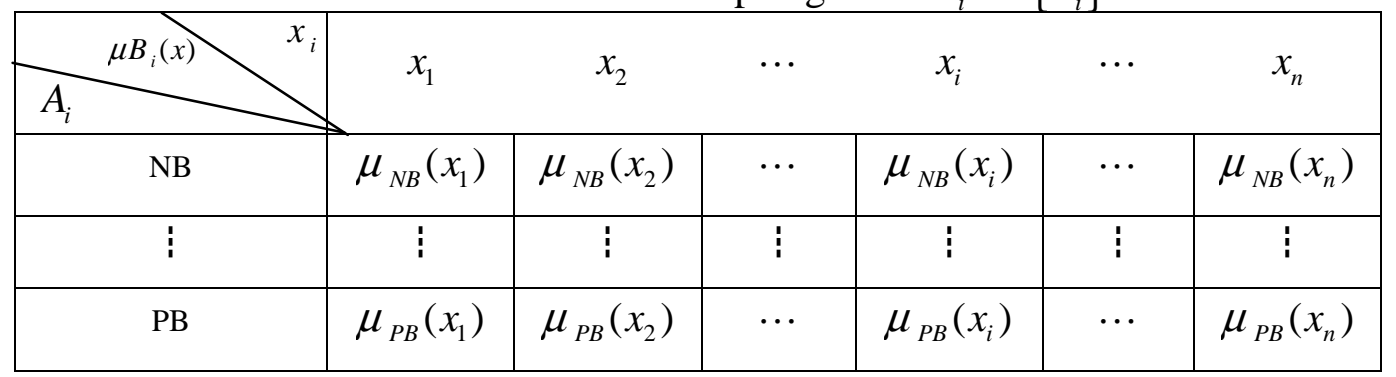

According to processing requirements, fuzzification $\mathrm{X}, \mathrm{Y}$, the fuzzy subset is shown in Fig.3, 4:

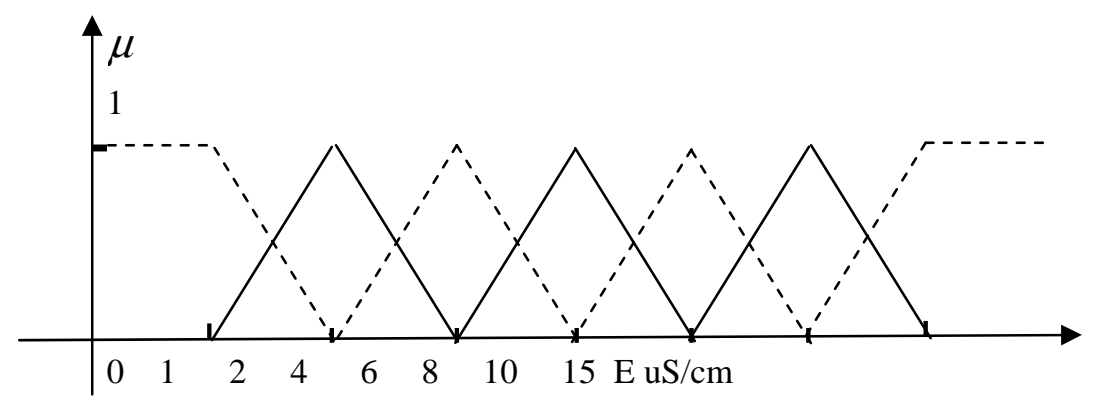

Fig. 3 The fuzzy subset of deviation of the current measurement values and required values of conductivity 


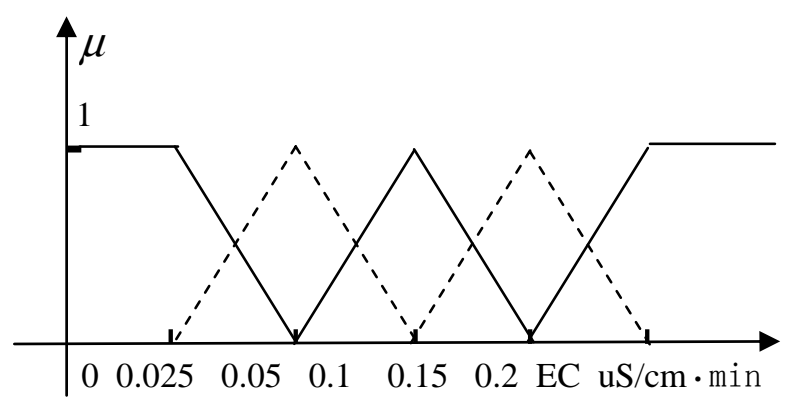

Fig. 4 The fuzzy subset of deviation change rate of the current measurement values and required values of conductivity

Let $\mu$ be the controlled variable of actuator (ion pump) at the processing stage, the corresponding domain: $Z=\left\{C_{k}\right\} \quad(k=1,2, \cdots, l)$. According to the operating experience, set the fuzzy control rules for: If $A_{i}$ then if $B_{j}$ then $C_{i j}$, its fuzzy relationship: $R=\bigcup_{i, j}\left(A_{i} \times B_{j} \times C_{i j}\right)$, namely, $\mu_{R}(x, y, z)=\vee_{i, j}\left(\mu A_{i}(x) \wedge \mu B_{j}(y) \wedge \mu C_{i j}(z)\right)$. When given $\mathrm{x}=A_{i}$ and $\mathrm{y}=B_{j}$, reasoning by the fuzzy composition rules: $C_{i j}=\left(A_{i} \times B_{j}\right) \circ R$, namely: $\mu_{C_{i j}}(z)=\underset{i, j}{\vee}\left[\left(\mu A_{i}(x) \wedge \mu B_{j}(y)\right) \wedge \mu_{R}(x, y, z)\right]$. Judgment using the maximum membership, determined output $\mu$ by the fuzzy subset $C_{i j}$.

Generate a control output lookup table, enter lookup table into the control software, directly lookup table to get $\mathrm{z}$ at real-time control. Obtained from the fuzzy control table is the control amount fuzzy level $\mathrm{z}$, when actually control actuator motor, $\mathrm{z}$ should be multiplied by the scaling factor $\mathrm{k}$, then for comparison with the set steady-state output values in order to avoid the conductivity increased during normal processing, if the former is bigger, then the fuzzy output value is taken as final output value, otherwise the steady-state output value as the final output value.

\section{Conclusion}

The combination of WEDM processing characteristics of the working fluid conductivity, fuzzy control rules of each fuzzy variable are set up and fuzzy control algorithm implemented. Fuzzy variables were used to describe the conductivity of the working fluid and processing material thickness and the electrode wire diameter. The actual processing shows that the working fluid conductivity control is more suitable for the use of fuzzy control method.

\section{References}

[1] Z.Y. Wang: WEDM process (Atomic Energy Press, Beijing 1998).

[2] N. Zhu, Q.P. Xu, W.G. Zhu: EDM and mold, Vol. 3, (2008), p.59.

[3] Z.X. Cai: Intelligent control theory and application (Tsinghua University Press, Beijing 2007).

[4] J. Zhu: Fuzzy control theory and system theory (Mechanical Industry Press, Beijing 2005).

[5] Mettler - Toledo Instruments (Shanghai) Co., Ltd. Measurement methods of solution conductivity, China, CN101135705, 2008.03.05.

[6] Wang M: Measurement Science \& Technology, Vol. 32, (2002), p.211. 